\title{
Sphingomonas paucimobilis
}

National Cancer Institute

\section{Source}

National Cancer Institute. Sphingomonas paucimobilis. NCI Thesaurus. Code C86749.

A species of strictly aerobic, Gram negative, rod shaped bacteria assigned to the phylum Proteobacteria. This species contains glycosphing olipids instead of lipopolysaccharide in its cell envelopes and can degrade lignan related biphenyls. $S$ paucimobilis is a rare human pathogen, and has been reported to cause bacteremia, ulcers, peritonitis, brain abscess, cervical adenopathy, splenic abscess, respiratory infection, urinary infection, and mening itis. 